\title{
The Contributions of African Traditional Medicine to Nigeria's Health Care Delivery System.
}

\author{
Ekeopara, Chike Augustine Ph.D ${ }^{1}$, Rev. Ugoha, Azubuike M.I ${ }^{2}$ \\ ${ }^{I}$ Department of Religious and Cultural studies University of Calabar Calabar - Nigeria \\ ${ }^{2}$ Department of Religious and Cultural Studies University of Calabar Calabar-Nigeria
}

\begin{abstract}
In recent times, traditional medicine has continued to receive increasing acceptance in Nigeria among many other African nations. The World Health Organization (WHO) estimated that about $80 \%$ of Asia, Latin America and African populations use traditional medicine to meet their primary health care needs. For a lot of people in these countries especially those living in rural areas, traditional medicines are the only available, easily accessible and affordable source of health care. This paper has tried to trace the historical background of traditional medicine in Nigeria, its contributions and progress made so far in health care delivery. It also considered various types of traditional medical services as well as methods of healing employed by traditional medical practitioners in reaching out to their numerous patients. This paper adopted qualitative research method in its approach. Qualitative research approach permits the researcher to investigate the issues that enable decision-making behaviour in a non-numerical manner. However, due to the nature of the research, oral and group interviews were added to elicit data from respondents. Research questions were also framed to elicit information from respondents using open-ended questions. The result of the integrated content analysis shows that despite various challenges facing traditional medicine, it has contributed immensely to the development of health care delivery system in Nigeria. The paper therefore, recommends among others, that in order to adequately maximize the health-care coverage of the multi-populated traditional Nigerian villages and communities with varied and variegated health challenges, sicknesses and ailments, there is obvious need for the formalization of the traditional health care services through the integration of traditional medicine into the health care delivery systems of various local and state governments in Nigeria.
\end{abstract}

Keywords: African traditional religion; traditional medicine; healthcare delivery

\section{INTRODUCTION}

In many developing countries especially in Africa, traditional medicine is still the main source of health care delivery in spite of the growth of religious enlightenment and western civilization in the areas of modern technology and orthodox medicine. According to experts, the World Health Organization records noted that an average of $80 \%$ of the total populations of Asia, Latin-America and Africa use traditional medicine in meeting their primary health care needs. This is as a result of what Kasilo and Trapsida (25) notes as "its cultural acceptability, affordability, and accessibility". For many people in Africa, Asia, Latin and America particularly and those living in rural areas, traditional/herbal medicine is the only available, accessible and affordable source of health care. There has been a steady upsurge of interest in the use of traditional medicine both in developed countries and developing countries of Africa, Asia, Europe and Latin America. In these countries herbal medicine is usually referred to as complementary and alternative medicine.

\section{DEFINITION}

According to the New International Webster Comprehensive Dictionary of English Language, Deluxe Encyclopedic Edition, the word traditional means "relating or depending on tradition" while tradition itself means "the transmission of knowledge, opinions, doctrines, customs, practices from generation to generation originally by word of mouth and by example (1330). Aso, it defined the word medicine as "a substance possessing or reputed to possess curative or remedial properties, the healing arts; the science of the preservation of health and of treating diseases for the purpose of cure" (791).

Health is a level of functional and/or metabolic efficiency of an organism, often implicitly human (Kasilo 33). In his own view Awofeso (I) defines health as "a dynamic state of well-being characterized by a physical and mental potential, which satisfies the demands of life commensurate with age, culture and personal responsibility," while Saracchi notes that health is "a condition of well-being free of disease or infirmity, and a basic universal human right" (33). However, Onunwa faulted the definition of Saracchi when he notes that:

Health as understood by Africans, particularly the Igbo of Nigeria, is far more social than biological. It does not entirely mean an absence of physical ailments. There is a clear unity of concept of psychosomatic 
interrelationships that is apparent reciprocity between mind and matter. Health therefore, is not an isolated phenomenon but part of the entire magico-religious fabric far more than an absence of disease (81).

Concurring to Onunwa's assertion, Australian Aboriginal people are of the view that "health does not just mean the physical well-being of the individual but refers to the social, emotional, spiritual and cultural wellbeing of the whole community". This is a holistic view of life and includes the cyclical concept of life and death. Nevertheless, the most common definition of health is the one given by World Health Organization (WHO) which sees it as "a state of complete physical, mental and social well-heing and not merely the absence of disease or infirmity" (134). The World Health Organization constitution also states that the enjoyment of the highest attainable standard of health is one of the fundamental human rights of every human being. Overall health is therefore achieved as a combination of physical, mental, emotional and social well-being which together is commonly referred to as the health triangle.

In the article, "Promoting the Role of Traditional Medicine in Health Systems: A strategy for the African Region", the World Health Organization summarized the definition of traditional medicine is the "sum total of all knowledge and practices, whether explicable or not, used in diagnosis, prevention and elimination of physical, mental or social imbalance and relying exclusively on practical experience and observations handed down from generation to generation, whether verbally or in writing". With these descriptions, various forms of medicines and therapies such as herbal medicine, massage, homeopathy, mud bath, music therapy, wax bath, reflexology, dance therapy, hydrotherapy, mind and spirit therapies, self-exercise therapies radiation and vibration, osteopathy, chiropractice, aromatherapy, preventive medicine, radiant heat therapy, therapeutic fasting and dieting spinal manipulation, psychotherapy, etc. are a few elements of traditional medicine (8). To Ekeopara, Traditional healing refers to all the methods that are employed for the cure and prevention of diseases and protection and preservation of lives and property of individuals against powerful forces of nature that are believed to pervade the environment (53). Adesina defines "traditional medicine as a cultural gem of various communities around the world and encompasses all kinds of folk medicine, unconventional medicine and indeed any kind of therapeutically method that had been handed down by the tradition of a community or ethnic group" (1). This is the understanding of traditional medicine that will undergird this work.

\section{RESEARCH QUESTIONS}

Since the researchers have adopted oral interview in elucidating information from respondents the following open-ended questions were asked selected respondents who practice traditional medicine in some parts of Nigeria.

1. What is your name?

2. How old are you?

3. What informed your choice of traditional medicine as an occupation?

4. How did you acquire your knowledge of traditional medicine and the relevant herbs and medicinal plants that you use?

5. How long have you been in the practice?

6. What is the level of your patronage?

7. What do you think is the reason behind your high patronage?

8. How effective and efficient is your medicine?

9. What types of ailments or sickness do you treat?

10. Do you think that your practice and medicine is contributing to the health care needs of members of your community?

The responses of the respondents were integrated into the research.

\section{RESEARCH METHOD}

The issue of research methodology is very crucial in a research of this nature. It is pertinent that a clear research method be articulated to enable a clearer understanding of why the authors arrived at the conclusion they did. There are two major research methodologies available for consideration in this work. The first is the qualitative research method which allows the researcher to explore the factors that contribute to decisionmaking behaviour in a non-numerical fashion. In this method, statistical analysis in not really considered but integrated content analysis is the main focus here and oral interviews and group discussions are used to gather data from respondents instead of questionnaires. The second research method is the quantitative research method which considers numerical figures from data in decision making. This method uses primary data such as questionnaires and various types of statistical analysis to arrive at its conclusion. In this research work on "The Contributions of Traditional Medicine to Nigeria's Health Care Delivery System", the authors have adopted qualitative research method. In this case content analysis of various authors has been adopted and personal and group interviews were made and the respondents' views were incorporated in the work. We did not adopt any form of statistical analysis in this work but used integrated content analysis. 
The Contributions of African Traditional Medicine to Nigeria's Health Care Delivery System.

\section{Origin of Traditional Medicine}

The origin and history of traditional medicine in Health Care Delivery as far as humanity is concerned is as old as the history of man himself. The Book of Beginnings recorded God as the manufacturer and originator of herbal medicine. And God Said, let the earth bring forth grass, the herb yielding seed, and the fruit tree yielding fruit after his kind, whose seed is in itself, upon the earth and it was so. And the earth brought forth grass, and herb yielding seed after his kind and the tree yield fruit, whose seed was in itself after his kind and God saw that it was good. (Genesis 1:10-11)

Before the creation of man, the Holy Book recorded that the Creator did not permit rain to fall upon the earth because there has not been created the man that will utilize the herbs that the earth will produce. After creating man, God made him the Estate Manager and Caretaker of after planting a botanical Garden in Eden. He told man that some of the fruits and herbs are good for food while others can be poisonous and are capable of causing death (Gen 2:5.7.8.9.15,16,17).

Attesting to the above assertion, Ekeopara in his book, African Traditional Religion, An Introduction, averred: It is quite probable that man as soon as he has reached the stage of reasoning found out through trial and error that plants can be used as food, that some might be poisonous and may even lead to death when eaten while some had medicinal value and power (52). The entire essence of traditional medicine revolves around the use of natural herbs in the bodily, spiritual, emotional and psychological healing of the total man. God is the source of nature and the manufacturer of the herbs. Therefore, God is the source of traditional or herbal medicine. King David in Psalm 104:14 stated thus: "He causes the grass to grow for the cattle and herbs for the service of man.

Onunwa (80), observed that healing is a part of the whole complex religious attempt by man to bring the physical and spiritual aspects of the universe as well as man who lives in it into the desired consistent harmony. The idea of "wholeness" is therefore not alien to the African mind. Healing becomes a cardinal religious practice because African cosmology demands that life in the world must be kept free from problem especially ill-health and obstacles that may hinder the fulfilment of desired goals. In the traditional African world view, health and healing are connected just in the same way they are with the fundamental theme of life. Herbs were the first medicines used by pre historic man. They are, therefore, part of every cultural tradition and have helped the development and growth of herbal medicine in Nigeria.

Still on the history of traditional medicine, Ekeopara noted that: Before the advent of western medicine our forebearers depended largely on traditional medicine and healing methods for the treatment of ailments and the cure of various types of diseases.

Mhame states that: Since the early 1970, the World Health Organization (WHO) has reportedly advocated for the recognition of Traditional Health Practitioners (THPs) as Primary Health Care Provider and for the integration of traditional medicine into the National Health System. Several calls have been made on Federal Government of Nigeria to take responsibility for the health of their people and to formulate national policies, regulations and standard, as part of comprehensive national health programs to ensure appropriate, safe and effective use of traditional medicine. (105)

Traditional medicine and its practitioners were officially recognized by the Alma Ata declaration in 1978 as an important resource for achieving the targeted goal of rendering good health care services for all by the year 2000. Since then, member States and the World Health Organization governing bodies have adopted a number of resolutions and declaration on traditional medicine. Notable among the resolutions are on "Promoting the Role of Traditional Medicine in Health Systems: A Strategy for the African Region". This resolution v as adopted by WHO Regional Committee for Africa in Ouagadougou, Burkina Faso, on August 31. 2000 and the declaration of the Decade of African Traditional Medicine (2000-20 10) by the Heads of State and Government in Lusaka, Zambia in July 2001. It was also resolved that a day be observed annually as African Traditional Medicine Day all over Africa and other parts of the world including Nigeria. That day is 31st day of August. The year 2010 marked a Decade since the institution of African Traditional Medicine Day. African Traditional Medicine Day was commemorated in 2003 in South Africa in conjunction with the 53 session of WHO Regional Committee for "African Traditional Medicine, our Culture, and Our Future" which Nigeria also attended. This singular act has created enabling environment for training and collaboration between practitioners of traditional medicine and conventional medicine for networking and information exchange.

\section{Types of Traditional Healers}

The traditional healer, as defined by the WHO (1976), is a person who is recognized by the community in which he lives as competent to provide health care by using vegetable, animal and mineral substances and certain other methods based on the social, cultural and religious background, as well as on the knowledge, attributes and beliefs that are prevalent in the community, regarding physical, mental and social well-being and the causation of disease and disability the following are the different types of healers in traditional African society: 
- Traditional Herbalists: Herbalists use mainly herbs, that is, medicinal plants or parts of such plants-whole root, stem, leaves, stem bark or root bark, flowers, fruits, seeds, but sometimes animal parts, small whole animal - snails, snakes, chameleons, tortoises, lizards, etc: inorganic residues -alum, camphor, salt, etc and insects, bees, black ants etc. is such herbal preparations may be offered in the form of (i) powder, which could be swallowed or taken with pap (cold or hot) or any drink, (ii) powder, rubbed into cuts or incisions made on an part of the body with a sharp knife, (iii) preparation, soaked for some time in water or local gin, decanted as required before drinking; the materials could also be boiled- in water, cooled and strained (iv) preparation pounded with native soap and used for bathing; such 'medicated soaps' are commonly used for skin diseases, (v) pastes, pomades or ointments, in a medium of palm oil or shea butter, or (vi) soup which is consumed by the patient. The herbalist cures mainly with plants which he gathers fresh. When seasonal plants have to be used, these plants are collected when available and are preserved usually by drying to eliminate moisture.

- Traditional Birth Attendants (TBAs): The World Health Organization defines a traditional birth attendant (TBA) as a person who assists the mother at childbirth and who initially acquired her skills delivering babies by herself or by working with other birth attendants. In the northern parts of the country, TBAs are of the female sex only, whereas in some other parts both males and females are involved. TBAs occupy a prominent position in Nigeria today as between 60-8 5 per cent of births delivered in the country and especially in the rural communities are by the TBAs.

They know how to diagnose pregnancy, confirm it and determine the position of the growing foetus. They have been seen to provide pre-natal and postnatal care and so combine successfully the duties of the modern-day mid-wife. Highly experienced TBAs have been recognized to assist in obstetric and Paediatrics care, as they manage simple maternal and hah hood illnesses. As a result of their exposure and experience, and more particularly the TBA's concept of human reproduction, as exemplified by pregnancy and childbirth being normal biological functions of human life linked holistically to cultural/social practices, TBAs have been trained to assist in orthodox' medicine practices at the primary health care level.

With their extra hands, a greater coverage of primary health care leading to improved maternal or child health and the lowering of maternal and child mortality and morbidity, have been achieved. TBAs are usually old and experienced women who see their assignments primarily as contributing their skill for the good of the community. With experienced TBAs, child delivery by Caesarean section is not common since it is hardly necessary to seek surgical help during child birth.

3. Traditional Surgeons: The various forms of surgery recognised in traditional medical care include: (i) the cutting of tribal marks: traditional surgeons usually cut tribal marks into the cheeks, bellies, etc. and charred herbal products are usually rubbed into these bleeding marks to effect healing,(ii) male and female circumcision (Clitoridectomy): traditional surgeons carry out these simple surgical operations with special knives and scissors: blood-letting operations and wounds that result from these operations are usually treated with snail body fluid or pastes prepared from plants.

These practices are, however, fast dying out in urban areas; (iii) removal of whitlow: diseased toes or fingers are usually cut open and treated. Piercing of ear lobes: particularly in the youth to allow the fixing of ear rings. Extraction of tooth: infected teeth or teeth with holes, that bring pain to the mouth are removed and treated with herbal medicines prepared in local gin.

4. Traditional Medicinal Ingredient Dealers: These dealers, more often women, are involved in buying and selling of plants, animals and insects, and minerals used in making herbal preparations. Some of them, who indulge in preparing herbal concoctions or decoctions for the management or cure of febrile conditions in children or some other diseases of women and children, may qualify to be referred to as traditional healers.

5. Traditional Psychiatrists: The traditional psychiatrist specializes mainly in the treatment of lunatics and those with mental disorders. Lunatics are usually restrained from going violent by chaining them with iron or by clamping them down with wooden shackles. People $\mathrm{w}$ ith mental disorders who are violent, particularly those that are demon possessed, are usually called or beaten to submission and then given herbal hypnotics or highly sedative herbal potions to calm them, in order to bring them to a state of mental, emotional and psychological calmness as well as tranquility. Treatment and rehabilitation of people with mental disorders usually take long periods.

Unlike the bone setter, the traditional psychiatrist and the traditional birth attendant whose duties are well defined and specialized, the herbalist is the general practitioner in traditional medicine. He is expected to be knowledgeable in all the various aspects of healing and in the functioning of the various organs of the body. Much is expected of him, as by his wealth of experience and knowledge he is expected to determine the nature of the patient's illness, treat him and also predict the course of his treatment. In a typical traditional setting, he corn bines the role of the present day doctor with that of the pharmacist and the nurse.

6. Practitioners of Therapeutic Spiritism: These practitioners include diviners or fortune tellers, who may be seers, alfas and priests, and use supernatural or mysterious forces, incantations, may prescribe rituals associated with the community's religious worship and adopt all sorts of inexplicable things to treat various 
diseases. The practitioners are usually consulted for diagnosis of diseases, their causes and treatment. With their ability to deal with the unseen, and the supernatural, they are usually held in high esteem in the community. They are believed to have extra-sensory perception and can see beyond the ordinary man. They can receive telepathic messages, can consult oracles, spirit guides etc. and perform well where other traditional healers and orthodox doctors fail. Their activities include, making prayers, citing and singing of incantations, making invocations and preparing sacrificial materials to appease unknown gods. It is believed that diseases which are caused by supernatural forces will be readily diagnosed and treated by these practitioners. This, in itself stems from the belief that certain medical ingredients - unusually large trees that are believed to house spirits, astronomic herbs, grave-yard plants like the physic nut, protective plants such as the wild colocynth or Sodom apple or even some reproductive herbs like the sausage tree or the tree of life itself- have spiritual powers and can be effectively utilized by these practitioners for the good of all.

The instructions which these practitioners use include magic stones which are usually thrown to the ground. Sounds so produced are read and interpreted. Some take replies of messages in a pool or glass of water. Others depend on the throwing of cowries, coins, kola-nut seeds, divining rods, keys or sticks, etc. Divination has come of age, its various functions linked with religion, creation myths, cosmology et cetera.

\section{Reasons for Growing Patronage for Traditional Medicine}

Health is the most precious of all things and it is the foundation of all happiness. Traditional medicine has developed in various communities in Nigeria in response to the health needs of the people. Many communities have since creation, developed various traditional systems using locally- available resources for the alleviation of their health poblem. As once noted some 13 years ago, traditional medicine is as old as the hills in Nigeria. The development of traditional medicine in Nigeria has led to the emergence of various categories of healers, the various healing methods, strategies and medicines or remedies now known. The British colonial masters brought in orthodox medicine and, today, both systems of medicine exist in the country; both have the primary objective to cure, manage or prevent diseases and maintain good health.

It is important to stress the relevance of traditional medicine to the majority of Nigerians. Most Nigerians, especially those living in rural communities don't have access to orthodox medicine and it is estimated that about 75 per cent of the populace still prefer to solve their health problems consulting traditional healers. Where such access exists, the rising cost of imported medications and other commodities used for medicines have posed a big problem. Besides, many rural communities have great faith in traditional medicine, particularly the inexplicable aspects as they believe that it is the wisdom of their fore-fathers which also recognizes their socio-cultural and religious background, which orthodox medicine seems to neglect. Recent reports show that more people in the world embrace traditional medicine.

To Ann Angbazo (1), several people have somewhat lost confidence in the efficacy of orthodox medicine. Analysts attribute the people's loss of confidence in orthodox medicine to factors such as drug counterfeiting and drug reactions, as well as the affordability and accessibility of the traditional healthcare delivery. They also observe that traditional medicine has been used to cure various life-threatening ailments in all parts of the world at lower costs, e en before the discovery of some medications. The general acceptance of traditional medicine is even attested to by the World Health Organization (WHO).

\section{Some Diseases and their Traditional Herbal Cures}

There is abundant justification for the use of herbs by the various traditional healers identified. Disease conditions identified in traditional medicine include:

(1) Cardiovascular disease hypertension, stroke etc. for which the antihypertensive herbs. the African Rauwolfia and the Negro coffee have been used to cure.

(2) Diseases of the nervous system-convulsions, insomnia etc. for which the parrot's beak and the African Rauwolfia also offer a good remedy.

(3) Diseases of the alimentary system-diarrhoea, dysentery etc. for which basil is useful.

(4) Diseases of the endocrine system-diabetes etc. for which the leaves of the common roused periwinkle or mormodica are valuable.

(5) Diseases of the respiratory system - asthma, cough etc. for which the lemon grass is of value.

(6) Diseases of the genital-urinary system, gonorrhoea, haematuria, etc. for which the bush banana is useful.

(7) Diseases of the skin-wounds, dermatonycosis etc. for which the craw-craw plant is very useful.

(8) Diseases associated with the ear, nose and throat ache, sinusitis etc. for which the resurrection plant is usually recommended and

(9) Diseases caused by microbes, viruses, insects etc. - infections, malaria etc. for which garlic, clove, the African mahogany etc. have been found useful. 
Numerous other diseases or complaints of a special nature such as hernia, snake bite, atiliritis, gout etc. have been treated using herbs alone or in admixture with animal parts and minerals. Today, plant medicines include vincristine and vinblastine isolated from the rose periinkle and used to treat childhood leukaemia and Hodgkin's disease, reserpine extracted from the African or Indian Rauwolfia and used in tranquillisers, diogenin extracted from the am and used in the treatment of rheumatism and to produce oral contraceptives and the shea butter hich showed nasal decongestant activity etc.

Non-plant medicines include the bee venom which is used in the treatment of arthritis and the cixet cat exudates which have shown anticonvulsant effects. Plant extracts and chemicals with muscle relaxant properties have been used by the TBAs to assist in child deli \cries. All these facts point to the values of medicinal plants and their importance in traditional medicine in present day Nigeria (Adesina 2).

\section{Contributions of Traditional Medicine to Healthcare Development}

It is an incontrovertible fact that traditional medicine has recorded meaningful and notable contributions which cannot be dismissed with a wave of the hand in Health Care Deliery Services not only in Nigeria alone but in many countries of Africa at large. Busia noted that:

Globally there is now a general recognition that traditional medicines, the medicines once described as primitive could be mankind's saving grace and therefore, within the past three decades, the changing view of herbs in particular as traditional medicine has moved from that of "witches brew" to major medicine (17).

It is estimated that out of a global population of 6.3.billon people in the world, about 4 billion people patronize the use of plants to meet their primary health care needs. It is also discovered that approximately about half the people living in urban cities in various states of Nigeria regularly make use of what is regarded as Complementary and Alternative Medicine. This increase in the demand and availability of services for Complementary Medicine has outpaced the developmental policy of the Federal Governments of Nigeria and the health sector.

- Remarkable Feats: In Nigeria, traditional medicine has been the main source of health care for the vast majority of people. It is currently estimated that between 70 and 80\% -Africans use traditional medicine for the management of both communicable and non communicable diseases such as cancer, malaria, HIV/AIDS, diabetes, Hypertension and tuberculosis. It is also noted that a high percentage of the rural populace patronize traditional midwifery for their maternal and neonatal health problems. Traditional Birth Attendants (TBA5) assists in majority of birth delivery of pregnant women in most Nigerian villages and communities.

Other areas where traditional medicine has contributed to the improvement of Health Care Delivery System includes the recent HI V/AIDS menace where Dr. Abalaka, discovered curative herbs for the dreaded disease which many patients have been reported treated on private consultation (Ekeopara 56). The disagreement he had with the Nigerian Medical Association has hindered the wider scope of beneficiaries of his discovery. Herbs are also used in quick healing of open injuries, stopping of bleeding, miscarriages, toothache, extraction of bullets from gun shots, etcetera.

- $\quad$ Primary Source of Modern Medicine: Kasilo (8), notes that "traditional Medicine has demonstrated great potential of therapeutic benefits in its contribution to modern medicine. An estimate of more than $30 \%$ of modern medicine is directly or indirectly sourced from traditional herbs such as analgesics (aspirin, Belladonna) anticancer medicines (Vincristine and Vinbiastine) anti-malaria (quinine, artemisinin); Anti-hypertensive agents (reserpine) and decongestants (ephedrine). As part of the contributions of traditional medicine in Health Care Delivery System in Nigeria, Ekeopara notes that:

...to make a barren woman productive, a special concoction specially prepared to cleanse the reproductive organs is administered to the woman three times daily for a period of three days. Rituals might accompany such administrations if it is believed that there is a spiritual agency involved in the barrenness (54).

This connotes that traditional medicine has a lot to still contribute today in treating barrenness among women.

- Manpower Coverage: In most Nigerian villages and communities, Traditional Health Practitioners (TI-IPs) generally are far more in number than doctors. In Nigeria and our neighbouring country, Ghana for example, statistics have shown that there are 25,000 and 10.000 patients for every medical doctor where as there are 200 and 100 patients respectively for $\mathrm{C} \backslash$ cry traditional Health practitioner. In view of the acute shortage of medical doctors in the country, it becomes an incontrovertible fact that Traditional Health Practitioners through traditional medicines have contributed immensely to the raising of manpower for healthcare coverage in Nigeria.

- Reduction of Child Mortality: A study carried out by WHO showed that in Ghana, Mali and Nigeria the first line of treatment for $60 \%$ of children with high fever resulting from malaria is the use of herbal medicines, (Busia 17). Going further in affirming the contributions of Traditional Medicine in Health Care Delivery, Obinna, making an overview of Traditional Medicine in Nigeria and other ECO WAS member States notes that 
at the 2009 African traditional medicine Day held in Lagos, the Lagos State Governor, Babatunde Fashola unequivocally opined that:

The pivotal role of traditional medicine in health care delivery in Africa and its potential to contribute to the attainment of the health-related provisions of the Millennium Development Goals MDGS) stressing that the reduction of child mortality, improving of maternal health and combating HIV/AIDS, malaria, tuberculosis, leprosies, child malnutrition, among to how far we are able to harness the hidden potential of our traditional medicine (17).

Commenting on the contributions of traditional medicine in Health Care Delivery System in Nigeria; Ekeopara commended:

...The Roman Catholic Church in Nigeria whose monks in Delta State, Nigeria, are devoting a lot of energy in research, in traditional medicine and healing. Their efforts have resulted in the production of various types of traditional medicine that are being patronized by many in Nigeria, for the cure of different ailments. These monks have resolved to find a cure for the dreaded human decimator and killer - HIV/AIDS. This effort should be encouraged and funded by the government and donor agencies for the overall development of traditional medicine and healing in Africa (57).

Onunwa (87), in his book Studies in Igbo Traditional Religion averred that in recent times, most of the practitioners of traditional healing have started to improve on their techniques, which includes the use of modern clinic system and introduction of some forms of new medication as an additional aid to the use of herbs and roots. In some of the traditional healing homes, the liquids extracted from leaves are stored in clean bottles, decently labeled and displayed on the shelves for patients to see and buy. He went further to state that: A lot of new things are happening in the traditional healing practices in recent times. A recent breakthrough claimed by one middle aged native doctor from Mbano in the South Igbo area is yet to be validated by modern research. The man, Dr. Njoku Nwigwe, did not only claim to have discovered how African herbs and roots could be used to cure stomach ulcers, pile and parasites in the human body without patients undergoing any surgical operation but also claimed to have made the discoveries known to the Chief Pharmacist in the Imo State Ministry of Health Owerri, Nigeria. The ministry is yet to confirm the validity of this claim (Onunwa 88).

If this is true it is a plus to traditional medicine.

\section{Different Traditional Health Care Services}

As part of its contribution to the development of Health Care Delivery Systems in Nigeria, traditional medicine has various Traditional Health Care Services through which it meets up with the health needs and challenges of the people. Busia (32) observes that, in African traditional medicine, the curative, training, primitive and rehabilitative services are referred to as clinical services. These traditional health care services are provided through tradition and culture prescribed under a particular philosophy. He went further to note that, the traditional norms, taboos and culture which are the bedrock of clinical practice of traditional medicine form the major reason for the acceptability of traditional health practitioners in their various communities of service (32).

The philosophical clinical care embedded in these guiding traditions, culture and taboos have contributed in no small measure in making traditional medicine acceptable and highly demanded by majority of the populace. These various health care services are:

Protective Services: A major part of African Traditional Medicine is for protective and preventive healthcare services. This guarantees spiritual and physical immunity from attack of physical sickness or spiritual forces. To this end Gelfand avers that: Roots, leaves or parts of animals or birds boiled in water or pulverized in fire from the basic ingredients of (traditional) medicine.., represent the major forces of nature. The vapour and smoke produced in boiling and pulverizing the medicines symbolize the air. In applying them to the human body, the link between nature and humanity is established in a very intense way and it generates power to protect and heal. The linkage is also implied in the use of charms and amulets which are medicines that protect (169).

This implies that traditional medicine has to do with nature healing.

- Curative Services: Most of the herbal medicines are also used to render curative ser ices for various ailments afflicting humankind. This is quite pivotal to the impact of traditional medicine in any given community.

- Destructive Services: There is element of evil in every good. There is also element of poison in every food. Traditional medicine even though it is used to render good services can as well be used to achieve evil motives. "There are medicines for good fortune, love, success, security of person and property... and there are also medicines against sorcery and witchcraft" (Evans-Pritchard 387). However, Magesa adds that, "significantly, all medicines contain all that ought to be used for the benefit of humanity, but can also be put to detrimental use by immoral individuals" (189).

- General Health Services: Kasilo (36), states that, general health services are services provided to clients by non-specialized health care providers. The general traditional health practitioner under general services can handle normal conditions like malaria, stomach upset, respiratory problems, rheumatism, arthritis, sexual dysfunctions, anemia and parasitic infections. 
- Mental Health Services: Traditional Medicine-men or Herbalists have proven to be very resourceful in rendering effective services to people with mental cases. They most often make use of divination to unravel the mental and psychological problems of their patients. DR Ination plays a key role in the treatment of neurosis and helps in re-tracing a patients' condition from its metaphysical past and influences it's interplay with the present and future.

- Midwifery Services: Midwifery is a health care profession in which providers give prenatal care to expecting mothers, attend to the birth of their infants and provide post-partum care to the mother and her infant (Mhame 37). Traditional Midwives are autonomous practitioners who are specialists in a low-risk pregnancy, childbirth and the post-partum stage. They help women to have healthy pregnancy and natural birth experience. They are trained to handle delivery situations that are also out of the norms.

In the African traditional setting, traditional midwives handle delivery cases intelligently. In the past 30 years, concerted efforts have been made in Nigeria to improve the kilIs and practices of traditional midwives, often referred to as Traditional Birth Attendants (TBAs). For the traditional midwives to be able to provide optimal care an enabling environment has to be provided and their collaboration with Orthodox nurses and doctors in health services strengthened (Busia 37).

- Bone Setting Services: A bone setter is a practitioner of joint manipulation. Before the advent of Chiropractors, Osteopaths and physical therapist and even establishment of orthopaedic hospitals, traditional bone setters were the main providers of this type of treatment. Bone setters do reduce joint dislocations and reset bone fractures (Busia 37). Many traditional bone setters make use of splints, bandages, plasters and clutches in the practice. They have been credited with success in treating very bad cases which were initially mismanaged in some orthopaedic hospitals. There are cases where orthopaedic surgeons had wanted to cut off a broken limb but when the relatives of the patients transferred them to the traditional bone setters, what was described as hopeless previously became treated by the traditional healers without amputation. Some physiotherapists have confessed that they on several occasions refer some cases to the traditional bone setters (Onunwa 88).

- Training and Promotional Services: The traditional medicine-men or herbalists engage themselves not only in ministering healing to the sick but also in rendering training services to young apprentices. Apprenticeship is a system or practice whereby training is given to a new generation of intending practitioners in order for them to acquire some skills to help in serving their communities. Training and promotional aspects of African traditional medicine services help to inculcate good characters in the practitioners which make them to be responsible. Accommodating, hardworking, good listeners as well as develop confidence in themselves, their traditions and cultural heritage.

- Rehabilitative Services: Traditionally, there is no system of skills development for disabled or physically challenged people in the Traditional African Community which would lead to their public or self employment. Each family or community in the spirit of "being your brother's keeper rather than brother's killer", helps in the rehabilitation of their own disabled persons. This social communal assistance which is called "Ubuntu philosophy" gives the disabled a sense of belonging creating an accommodating way of living through tradition, culture, norms and taboos (Mhame 38). Every disabled person therefore is accepted and recognized as part of the family or community and is supported to lead a functional and fulfilled life in spite of his apparent disability. With this kind of communal social support every physically challenged person discovers ability in his disability. This brings about mental, emotional and psychological healing to the physically challenged person.

\section{Methods of Traditional Healing}

There are various methodological processes involved in the administration of traditional medicine in Nigeria through which the goal of restoration of human health is achieved. They are namely:

- Knowledge of Herbs: The major method in the administration of traditional healing via local herbs is through a good knowledge of herbs and roots and the right application of the same to the patients who need them. Onunwa notes that treatment most times commences with simple herbs and antidotes which may be administered for one day or two. He further stated that the art of healing has been traditionally associated with the thorough knowledge of the use of herbs and roots and the appropriate rituals to perform at the appropriate time (82). Kasilo (89) noted that the traditional knowledge of herbs has played a significant role in the healthcare systems in Nigeria and other countries of the African Region for centuries. Traditional medicines are presently used by nearly $80 \%$ of the population. Owing to the global resurgence in the use of natural products and the advent of the biotechnological industry, traditional knowledge is increasingly becoming a source of modern drug development and biotechnological inventions. Despite the important role of traditional knowledge, traditional communities are unable to protect their knowledge through the existing intellectual property system owing to the failure of the knowledge to satisfy the requirements for intellectual property protection. Parrinder (56), in his book West African Religion, observed that, "West African doctors have a wide knowledge of the properties of many roots, barks, leaves, and herbs. They are called 'observers' of plants in Fon and workers in roots in Furi". 
It is also noted that:

The traditional medicine men are not only knowledgeable in the variety of roots and herbs that have therapeutic values, but also understand the psychology of their people's values. Their knowledge of the healing powers in roots and leaves is amazing. (Onunwa 87)

- Divination: When the condition of the patient is not improved after the application of simple treatments, the method of divination is applied whereby a diviner could be invited for spiritual guidance as to ascertain the remote and esoteric cause of the protracted ailment. The diviner may also recommend the type of medicine to be applied for quick cure. Such recommendations may also involve rituals.

- Ritual Washing: Another method of traditional healing is through ritual cleansing. This is carried out by the traditional healer commonly called medicine-man on the patient. This is done purposely to appease the deities and ancestors and restore a good and cordial relationship between the patient and the deities so that their goodwill will cause any medicine applied in making him to recover to be effective. To Onunwa,

the hospital is believed to be good for certain diseases whose treatment must however, be validated by some ritual offerings at home. This is based on the people's world view. In spite of the contributions of modern medicine, some traditional lgbo men still believe that healing is incomplete if some necessary rituals are not performed. They do not completely despise the achievements of Western medicine, but believe that there are some ailments which the hospitals cannot cure (90).

iv Diagnosis vaetiology: Common ailments or problems are easily recognised and treated successfully, based on the symptomatology of the disease. If the illness persists, recourse is made to the practitioner of occultism/the diviner who' in his efforts to discover the real cause of the disease, may consult various spirits including his oracle, pre scribe appropriate rituals and make sacrifices to solicit the right answer. This is because they believe that diseases can be caused by sorcery, ghosts, breach of taboo, spirit intrusion and acts of the Supreme Being. It has also been found that public opinion and rumours may help the diviner to trace the cause of a disease. Diagnosis may also be achieved by visual, examination of eyes, skin, urine, faeces etc.; taste, for example, of urine for sugar in the diabetic, use of ants to detect sugar in the urine of the diabetic, palpation that is through the sense of touch using palms, fingers or the analysis of a patient's dreams. Proper diagnosis is needed in the treatment of psychosomatic and psychiatric cases just as in the eases of bone fractures and other traumatic injuries. Generally, proper diagnosis is the key to most traditional treatments.

v. Symptom Analysis: The patient describes the symptoms as much as practicable. The healer also watches out for these as well as analyses them and from the careful observations make his prescriptions. For the various diseases, symptoms that are usually watched out for include behavioural changes; fever or abnormal rise in body temperature; jaundiced state, yellow colour in the eyes, urine, palm and fingers suggesting possible lier problems; seizures as often experienced in convulsions, diarrhoea in cases of poisoning; frequent stooling and vomiting; bleeding which may be from the nose, mouth, gums, teeth, from private parts, anus; blood in the urine; coughing and vomiting blood; breathing difficulty; sneezing; swelling which may be minor and localised; general weakness of the body; and development of rashes and even pain. Experienced herbalists can readily differentiate constant or intermittent pains from sharp or dull ones and link them with specific diseases. Signs: These are usually observed by experienced healers. Modern-day healers are being encouraged to use stethoscopes and other simple instruments to determine signs.

vi. Treatment: The herbalist treats with medicines made up of plants, animals and minerals. He may also use any of the therapeutic systems described earlier such as fasting and dieting massage, therapeutic spiritism et cetera.

\section{Challenges Facing Traditional Medicine}

There are myriads of serious challenges militating against the progress and development of Traditional Medical practice in Nigeria. Although a lot of progress has been made in implementing the regional strategy on promoting the role of traditional medicine in Health Care System, the Nigerian state has continued to face some challenges that hamper the institutionalization of traditional medicine into our National Health Care Systems. These challenges include:

- Poor Organizational Arrangement: There is limited Organizational arrangement for the institutionalization of traditional medicine such as:

- Poor allocation of financial resources for implementation of traditional medicine activities

- Delay in the establishment of mechanisms for the official recognition of traditional health practitioners.

- Lack of national policies in the country

- Limited national strategic plans for policy implementation

- Lack of mechanisms of collaboration between practitioners of conventional and traditional medicine.

- Limited Research Data: There is also a challenge of limited research data on the safety, efficacy and quality of traditional medicines and documentation of traditional medicine practices. 
- Oral Tradition/Documentation: The oral nature of the knowledge of traditional medicinal herbs transmitted in various forms from one generation to another without proper documentation poses a serious challenge in the preservation of such knowledge.

- Non Inclusion in School Curriculum: Majority of the States and Local Government

Councils are yet to include some aspects of traditional medicine in the curricula of Health Science Students and other institutions of higher learning.

- Lack of National Policy: Majority of countries are yet to develop National Policies on the conservation of medicinal plants and get engaged in large-scale cultivation of medicinal plants in botanical gardens.

- Quality Control: There is the challenge of ensuring the safety and efficacy of herbal medicines, the quality of the source of raw materials, cultivation and harvesting, field collection, transport and storage, correct identification of species of medicinal plants.

- Effective Monitoring: Adverse events arising from consumption of herbal medicines calls for effective monitoring. The issue of misidentification, adulteration, wrong labeling, contamination with toxic substances, over dosage, misuse of herbal medicines $\mathrm{H}$ both health-care providers and consumers and the concomitant use of herbal medicine at the same time with other orthodox medicines.

- Lack of Knowledge: There is challenge of lack of knowledge of herbal medicine by Health Care regulatory authorities and agencies.

- Conservation/Preservation: Ernest Rukangira (38), observed that African medicinal plant resources may be doomed to extinction by overexploitation resulting from excessive commercialization, habitat destruction and other natural and man-made destructive influence unless serious conservation measures are taken to ensure their continued availability. This can be done through establishment of medicinal plant garden and farms.

\section{The Way Forward}

If all the healing and curative treasures divinely embedded in our medicinal plant by the Mother Nature (the Supreme Being) will be adequately maximized in improving the health services being rendered to our people, definite and decisive actions need to be taken. These include:

- Research: Research in traditional medicine and development need to be included in the national health research agenda.

- Curriculum: Traditional Medicine should be included in the curricula of our primary, secondary and tertiary institutions.

- Collaboration: There should be a forum and enabling environment provided for effective collaboration between Orthodox Medical and Traditional Medicinal Practitioners. The gulf existing between them which give room for jealousies and destructive criticisms should be narrowed.

- Protection: Mechanisms for the protection of intellectual property rights and indigenous herbal knowledge should be developed.

- Training: Establishment of regular training programmes or National Institute for Traditional or Alternative Medicine, for traditional medicine practitioners should be encouraged.

- Budget: Countries should be encouraged to create a budgetary allocation for traditional medicine in their National Health Budget and include the expenditure of traditional medicinal care in National Health Accounts.

- Legislation: Legislative framework and national policy for the protection of the knowledge of medicinal plants as well as it preservation from wanton destruction through reckless bush burning and tree-cutting should be put in place.

- Documentation: Government should establish units or departments for formal documentation of the details of the working of medicinal plants from the existing oral tradition in order to guard against its extinction or possible adulteration.

- Packaging \& Marketing: The Federal Government and the Federal Ministry of Health should establish governmental agencies that will take care of both the packaging and the effective marketing of the traditional herbals productions both within and outside the country. A Department of Traditional/Alternative medicine should be created in all Health Ministries at all levels of government.

- Trade Fairs and Exhibition: Annual National and International Trade Fairs and Exhibition on traditional medicines should be organized and co-sponsored by government and non-governmental agencies, philanthropists and various stakeholders. This should be institutionalized as it has already commenced.

Mrs Julie Offoboche, a former Solicitor-General of Cross River State, urged the Federal Government of Nigeria to acknowledge the work of traditional medicine practitioners by licensing them and creating room for them in hospitals. She stressed that although orthodox medicine has a great value in healthcare delivery, there were some ailments that could only be effectively treated with local medicinal herbs. Offoboche notes that valuable goods and services, including herbal medicine, were being produced in Nigeria, urging the government to give tangible support to these small-scale enterprises. "The government should give them some grant and ensure that the amount of money given to them is actually given to the right persons". When this is done, herbal 
medicine practitioners would be independent and be able to give quality service delivery; they can even perform better than their counterparts in India and China. As regards herbal products coming in from India and China, people will tell you I took this and I took that but it did not work; when they take the Nigerian herbal formula, it would work for them. Our herbal products are very efficacious. The government should give support to the herbal medications' producers by giving them grants to enable them to conduct research and produce more drugs," she added. Nevertheless, Offoboche notes that the Cross River State Government had been licensing herbal medicine practitioners via annual renewable agreements, urging the Federal Government to replicate the policy at the federal level.

\section{EVALUATION AND CONCLUSION}

The African philosophy of clinical health care practice is a bridge-builder existing between people's well-being and enjoyment of the goodness of life graciously given to us by our creator and the Supreme Being. Therefore, in order to adequately maximize the health-care coverage of the multi-populated traditional Nigerian villages and communities with varied and variegated health challenges, sickness and ailments, there is obvious need for the formalization of the traditional health care services through the integration of traditional medicine into the health care delivery systems of various Local and State Governments in Nigeria.

There is also need for enhanced collaboration between practitioners of orthodox conventional medicine and traditional medicine for the benefit of all. The earlier this is done, the better for all of us. However, there is no gain saying that in spite of criticism, spite and distaste for traditional healing, the fact remains that many Africans do not hesitate to seek the help of traditional medicine men/women when confronted with life threatening situations or problems. Evidence abounds as to the veracity of this assertion. Orthodox doctors have been known to refer medical cases beyond their comprehension to the traditional practitioners, especially when such medical cases could not be scientifically diagnosed. Traditional medicine is holistic, in the sense that it heals both physically and spiritually that is, it heals both body and soul. The world is yet to find a cure for HI V/AIDS. Perhaps the cure will be found if more energy and attention is directed to traditional healing methods (Ekeopara 57).

It is noteworthy to state, that despite the impact of western civilization, orthodox medicine and religious awakening/enlightenment, the typical African man seeks help from the gods of his fathers through traditional medicine whenever the chips are down. Idowu, concurred to this assertion when he stated in his book, African Traditional Religion: A Definition that it is well known that in strictly personal matters relating to the passages of life and the crisis of life, African Traditional Religion is regarded as the final succour by most Nigerians and an average African.

In hospitals for example, people who go on admission and have declared themselves Christians and indeed are practicing Christians, have medicines prepared in the traditional wav smuggled in to them simply because psychologically at least, that is more effective in that it is consecrated medicine with the touch of the divine in contrast to the European's mere lored water. In matters concerning providence, healing and general well-being therefore, most Africans still look up to their religion as the way (206). There is therefore a need for traditional medicine to be practiced as an alternative medicine to orthodox medicine.

\section{WORKS CITED}

[1]. Adesina. S.K. "Traditional Medical Healthcare in Nigeria". http: /www.google.com/search?hl=enGB\&redir.. .es... Retrieved February, 15 2013. Web. March, 132013

[2]. Anna. A. Incorporating Traditional Medicine Into Nigeria's Healthcare System" peoplessdailyng. com/incurprating-traditi... Retrieved on January 23, 2013. Web March 15, 2013.

[3]. Awofeso, N. "Re-defining Health" in an Article in: Ustim and Jakobi 2005; http ://www.who . int/bul letin/bulletin_board/83/ustin 11 05/en/print.html. Web, 28 June 2010.

[4]. Busia K. Medical Provision in Africa-Past and Present. Phototherapy Research, 2005. Health Monitor, a special issue 14. A serial publication of the World Health Organization Regional Office for Africa. ISSN 20776136, August, 2010.Print

[5]. Ekeopara. Chike Augustine. African Traditional Religion: An Introduction. Calabar: Natos Affair, 2005.Print.

[6]. Evans-Pritchard, E. E. Witchcraft, Oracles, and Magic among the Azande. Oxford: Clarendon Press, 1997. Print

[7]. Gelfand. M. Witch Doctor: Traditional Medicine-Man of Rhodesia. New York: Frederick Praeger, 1964. Print.

[8]. Idovu. E.B. African Traditional Religion: A Definition. London: S.C.M. Press Limited, 1973. Print.

[9]. Kasilo. Ossy MJ and Trapsida Jean-Marie. Health Monitor, a special issue 14. A serial publication of the World Health Organization Regional Office for Africa. ISSN 20776136, August, 2010. Print. 
[10]. Kasilo. Ossy MJ. "Clinical Practices of African Traditional Medicine" in Health Monitor, a Journal of the World Health Organization. Regional Office for Africa. A special issue. ISSN 2077 6138. August 2010.

[11]. Magesa. Laurenti. African Religion: The Moral Traditions of Abundant Nairobi: Paulines Publications Africa, 1998. Print.

[12]. Nlhame. Paulo Peter. "Clinical Practices of African Traditional Medicine" in Health Monitor. Special Issue 14. A serial Publication of the World Health Organization Regional Office for Africa. ISSN 20776136. August 2010. Print.

[13]. New International Webster Comprehensive Dictionary of the English Language, Deluxe Encyclopedia Edition. Naples Florida: Trident Press International, 2004. Print.

[14]. Obinna C. "Review of African Traditional Medicine, Roots and Rooted". http://www.rootsand rooted.org/?p=986. Web. 10 August 2009.

[15]. Onunwa. Udobata. Studies in Igbo Traditional Religion. Obosi: Pacific Publishers, 1990.

[16]. Parrinder, E.G. West African Religion. London: Epworth Press, 1959. Print.

[17]. "Promoting the Role of Traditional Medicine in Health System: A Strategy for the African Religions". World Health Organization, 2001. WHO Regional Office for Africa, Temporary Location. Harare, Zimbabwe. (Document AFR/Rc50/9 and Resolution A F R/Rc5 0/R3).

[18]. Rukangira. Ernest. Medicinal Plants and Traditional Medicine in Africa: Constraints and Challenges. Nairobi: Conserve Africa International.1996.

[19]. World Health Organization (1978). Alma-Ata Primary Health Care. World Health Organization. Geneva.

[20]. World Health Organization (1996): "Traditional Medicine". WHO Fact Sheet.

[21]. World Health Organization (WHO). Traditional Medicine Strategy 2002-2005. WHO, Geneva. WHO/EDM/TRM/2002. 1. 